\title{
Chapter 139 \\ Report on Global Environment Competitiveness of the United Arab Emirates
}

The United Arab Emirates is a country situated at the southeast of the Arabian Peninsula in Western Asia on the Persian Gulf, bordered by Oman to the east and Saudi Arabia to the south, and sharing sea borders with Qatar to the west and Iran to the north. It covers 83.6 thousand of square kilometers and has a population of 7.89 million.

\begin{tabular}{|l|}
\hline Score: \\
45 \\
\hline Rank: \\
114 \\
\hline
\end{tabular}
Its GDP reaches $\$ 360.25$ billion in 2011 . Through the index system and evaluation model, the environment competitiveness index of The United Arab Emirates ranks at 114 in 133 countries.

Fig. 139.1 Contribution

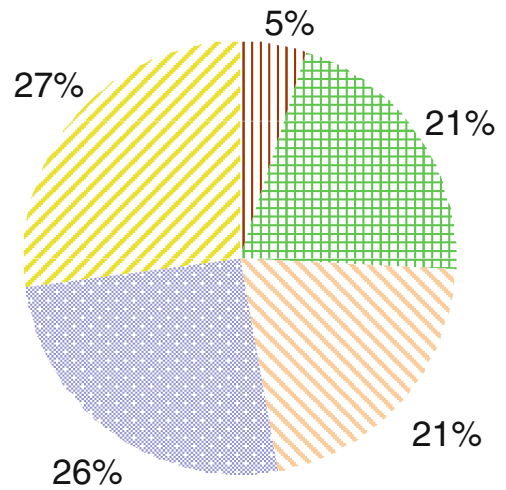

III Resource Environment Competitiveness

Ecological

Environment

Competitiveness

Environment

Carrying

Competitiveness

Environment

Management

Competitiveness

of sub-index to GEC

Environment

Harmony

Competitiveness 


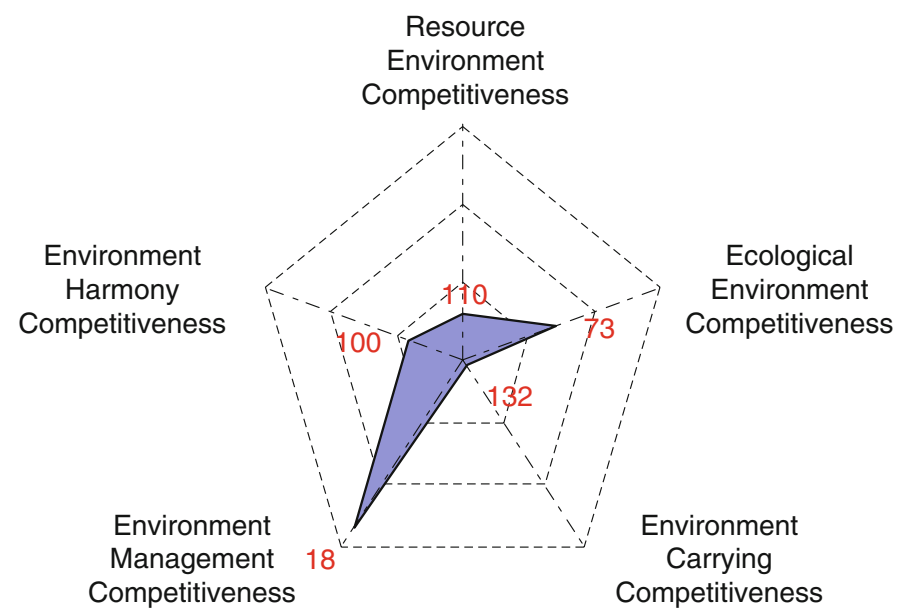

Fig. 139.2 Rank of sub-index of GEC

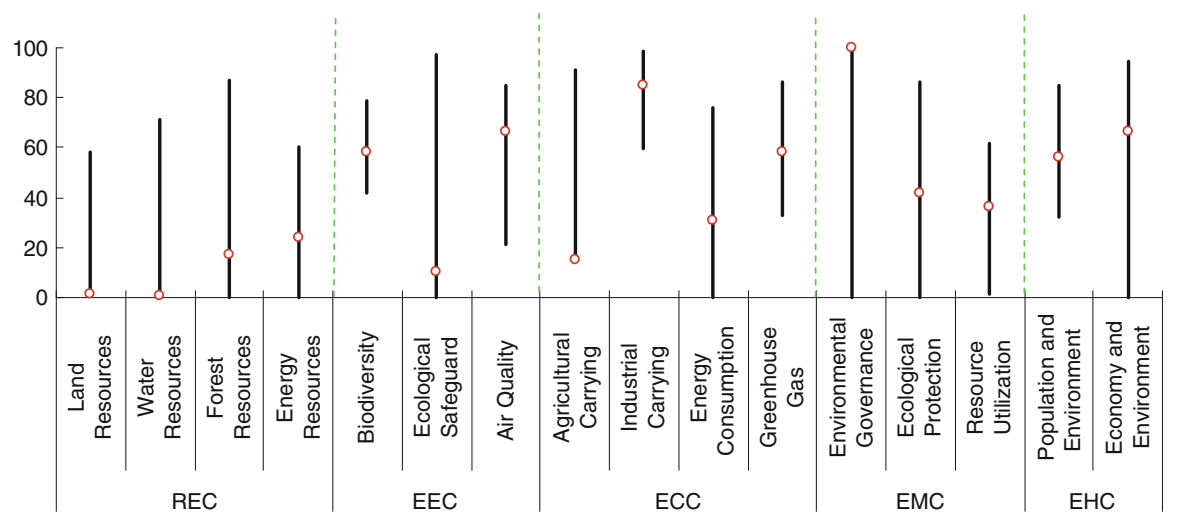

Fig. 139.3 Score and rank of the pillars of GEC

Table 139.1 Score and rank of all indicators of GEC

\begin{tabular}{|c|c|c|c|c|c|}
\hline Indicators & Score & Rank & Indicators & Score & Rank \\
\hline 1 Resource Environment & 10.98 & 110 & Groundwater & 0.16 & 121 \\
\hline Competitiveness & & & \multirow{2}{*}{$\begin{array}{l}\text { Total internal renewable } \\
\text { water resources }\end{array}$} & \multirow[t]{2}{*}{0.09} & \multirow[t]{2}{*}{129} \\
\hline 1.1 Land Resources & 1.10 & 131 & & & \\
\hline Land area per capita & 1.89 & 83 & 1.3 Forest Resources & 16.88 & 111 \\
\hline $\begin{array}{l}\text { Percentage of arable land } \\
\text { to total land area }\end{array}$ & 0.85 & 129 & $\begin{array}{l}\text { Growing stock in forest and } \\
\text { other wooded land }\end{array}$ & 50.05 & 105 \\
\hline Arable land per capita & 0.29 & 131 & \multirow{2}{*}{$\begin{array}{c}\text { Proportion of land area } \\
\text { covered by forest }\end{array}$} & \multirow[t]{2}{*}{4.46} & \multirow[t]{2}{*}{112} \\
\hline 1.2 Water Resources & 0.73 & 129 & & & \\
\hline Surface water & 0.02 & 124 & Forest area per capita & 0.28 & 113 \\
\hline Annual precipitation & 2.64 & 127 & 1.4 Energy Resources & 23.90 & 7 \\
\hline
\end{tabular}


Table 139.1 (continued)

\begin{tabular}{|c|c|c|c|c|c|}
\hline Indicators & Score & Rank & Indicators & Score & Rank \\
\hline Fossil energy & 33.83 & 4 & \multirow{2}{*}{$\begin{array}{l}\text { Energy consumption per unit } \\
\text { of land area }\end{array}$} & \multirow[t]{2}{*}{98.42} & \multirow[t]{2}{*}{115} \\
\hline Energy production & 23.71 & 6 & & & \\
\hline $\begin{array}{l}\text { Proportion of combustible } \\
\text { renewable and waste to }\end{array}$ & 0.04 & 116 & $\begin{array}{l}\text { Ratio of clean energy } \\
\text { consumption }\end{array}$ & 0.00 & 116 \\
\hline total energy consumption & & & Elasticity of energy & 13.83 & 73 \\
\hline $\begin{array}{l}\text { Net energy imports of the } \\
\text { energy consumption }\end{array}$ & 36.28 & 13 & $\begin{array}{l}\text { Elasticity of electric power } \\
\text { consumption }\end{array}$ & 11.55 & \multirow[t]{2}{*}{38} \\
\hline $\begin{array}{l}2 \text { Ecological Environment } \\
\text { Competitiveness }\end{array}$ & 47.08 & 73 & $\begin{array}{l}\text { consumption } \\
\text { 3.4 Greenhouse Gas }\end{array}$ & 57.96 & \\
\hline $\begin{array}{l}\text { 2.1 Biodiversity } \\
\text { Threatened fish species }\end{array}$ & $\begin{array}{l}58.09 \\
93.87\end{array}$ & $\begin{array}{l}66 \\
36\end{array}$ & $\begin{array}{l}\text { Growth rate of } \mathrm{CO}_{2} \\
\text { emissions }\end{array}$ & 53.77 & 57 \\
\hline $\begin{array}{l}\text { Threatened mammal species } \\
\text { Threatened plant species }\end{array}$ & $\begin{array}{r}96.20 \\
100.00\end{array}$ & $\begin{array}{r}37 \\
1\end{array}$ & $\begin{array}{l}\text { Growth rate of Methane } \\
\text { emissions }\end{array}$ & 51.21 & 107 \\
\hline $\begin{array}{l}\text { GEF benefits index for } \\
\text { biodiversity }\end{array}$ & 0.20 & 109 & $\begin{array}{l}\mathrm{CO}_{2} \text { emissions per unit of } \\
\text { land area }\end{array}$ & 97.95 & 121 \\
\hline 2.2 Ecological Safeguard & 10.30 & $\begin{array}{l}98 \\
93\end{array}$ & $\begin{array}{l}\mathrm{CO}_{2} \text { emissions per unit of } \\
\text { energy consumption }\end{array}$ & 33.09 & 95 \\
\hline Marine protected areas & $\begin{array}{r}14.93 \\
3.32\end{array}$ & 52 & $\begin{array}{l}4 \text { Environment Management } \\
\text { Competitiveness }\end{array}$ & 57.63 & 18 \\
\hline $\begin{array}{l}\text { 2.3 Air Quality } \\
\text { Inhalable particles (PM10) }\end{array}$ & $\begin{array}{l}66.41 \\
35.04\end{array}$ & $\begin{array}{r}53 \\
123\end{array}$ & \multirow{2}{*}{$\begin{array}{l}\text { 4.1 Environmental } \\
\text { Governance } \\
\text { Agricultural chemicals } \\
\text { regulation }\end{array}$} & 100.00 & 1 \\
\hline $\begin{array}{l}\text { Particulate matter (PM2.5) } \\
\text { Index of indoor air pollution }\end{array}$ & $\begin{array}{r}65.42 \\
100.00\end{array}$ & $\begin{array}{r}116 \\
1\end{array}$ & & N/A & N/A \\
\hline $\begin{array}{l}\text { Nitrogen oxides emission } \\
\text { Sulfur dioxide emission }\end{array}$ & $\begin{array}{l}68.76 \\
40.05\end{array}$ & $\begin{array}{l}22 \\
88\end{array}$ & \multirow{2}{*}{$\begin{array}{l}\text { Percentage of the rural } \\
\text { population with } \\
\text { access to an improved } \\
\text { water source }\end{array}$} & \multirow[t]{2}{*}{100.00} & \multirow[t]{2}{*}{1} \\
\hline $\begin{array}{l}3 \text { Environment Carrying } \\
\text { Competitiveness }\end{array}$ & 47.95 & 132 & & & \\
\hline 3.1 Agricultural Carrying & 15.29 & 132 & \multirow{3}{*}{$\begin{array}{l}\text { Percentage of the urban } \\
\text { population with } \\
\text { access to an improved } \\
\text { water source }\end{array}$} & \multirow{3}{*}{100.00} & \multirow[t]{3}{*}{1} \\
\hline $\begin{array}{l}\text { Cereal yield per unit of } \\
\text { arable land }\end{array}$ & 25.90 & 76 & & & \\
\hline $\begin{array}{l}\text { Fertilizer consumption } \\
\text { per unit of arable land }\end{array}$ & 16.43 & 128 & & & \\
\hline $\begin{array}{l}\text { Annual freshwater } \\
\quad \text { withdrawals for } \\
\text { agriculture per unit }\end{array}$ & 0.00 & 130 & $\begin{array}{l}\text { Area of plantation and } \\
\text { afforestation } \\
\text { Biome protect }\end{array}$ & 0.41 & 61 \\
\hline $\begin{array}{l}\text { of arable land } \\
\text { 3.2 Industrial Carrying }\end{array}$ & 85.26 & 110 & $\begin{array}{l}\text { Overfishing of fishing } \\
\text { resources }\end{array}$ & 51.52 & 53 \\
\hline $\begin{array}{l}\text { Net exports of goods as a } \\
\text { percentage of GDP }\end{array}$ & 44.87 & 131 & 4.3 Resource Utilization & $\begin{array}{l}36.51 \\
8243\end{array}$ & $\begin{array}{r}68 \\
2\end{array}$ \\
\hline Electric power consumption & 96.27 & 23 & $\begin{array}{l}\text { Utilization rate of water } \\
\text { resources }\end{array}$ & 82.43 & 2 \\
\hline $\begin{array}{l}\text { per unit of value added of } \\
\text { industry }\end{array}$ & & & $\begin{array}{l}\text { Percentage of total internal } \\
\text { renewable water }\end{array}$ & 55.56 & 81 \\
\hline $\begin{array}{l}\mathrm{SO}_{2} \text { emissions per unit of } \\
\text { value added of industry }\end{array}$ & 99.94 & 30 & $\begin{array}{l}\text { resources to total water } \\
\text { resources }\end{array}$ & & \\
\hline $\begin{array}{l}\text { Annual freshwater } \\
\text { withdrawals for industry }\end{array}$ & 99.97 & 6 & $\begin{array}{l}\text { Percentage of agricultural } \\
\text { land to total land area }\end{array}$ & 8.05 & 128 \\
\hline $\begin{array}{l}\text { per value added of } \\
\text { industry }\end{array}$ & & & Percentage of fossil fuel & 0.00 & 126 \\
\hline 3.3 Energy Consumption & 30.95 & 109 & total energy consumption & & \\
\hline
\end{tabular}


Table 139.1 (continued)

\begin{tabular}{|c|c|c|c|c|c|}
\hline \multirow{3}{*}{$\begin{array}{l}\text { Indicators } \\
\begin{array}{c}\text { Environment Harmony } \\
\text { Competitiveness }\end{array}\end{array}$} & \multirow{3}{*}{$\frac{\text { Score }}{61.38}$} & \multirow{3}{*}{$\frac{\text { Rank }}{100}$} & Indicators & \multirow{2}{*}{$\frac{\text { Score }}{44.49}$} & \multirow{2}{*}{$\begin{array}{l}\frac{\text { Rank }}{129} \\
\end{array}$} \\
\hline & & & $\mathrm{CO}_{2}$ emissions per capita & & \\
\hline & & & Energy consumption per & 35.77 & 123 \\
\hline 5.1 Population and & 56.07 & 123 & capita & & \\
\hline Environment & & & 5.2 Economy and & 66.69 & 54 \\
\hline Percentage of population & 97.00 & 39 & Environment & & \\
\hline $\begin{array}{l}\text { with access to Improved } \\
\text { sanitation facilities }\end{array}$ & & & $\begin{array}{l}\text { Land resource utilization } \\
\text { efficiency }\end{array}$ & 1.26 & 18 \\
\hline $\begin{array}{l}\text { Motor vehicles per } 1,000 \\
\text { people }\end{array}$ & 61.60 & 93 & $\begin{array}{l}\text { Sulfur dioxide emissions per } \\
\text { unit of GDP }\end{array}$ & 96.64 & 45 \\
\hline $\begin{array}{l}\text { Renewable internal } \\
\text { freshwater resources per }\end{array}$ & 0.02 & 132 & $\begin{array}{l}\text { Carbon dioxide emissions } \\
\text { per unit of GDP }\end{array}$ & 81.84 & 87 \\
\hline $\begin{array}{l}\text { capita } \\
\mathrm{SO}_{2} \text { emissions per capita }\end{array}$ & 82.04 & 110 & $\begin{array}{l}\text { Energy consumption per unit } \\
\text { of GDP }\end{array}$ & 87.04 & 57 \\
\hline
\end{tabular}

Table 139.2 Rank distribution of the individual indicators of GEC

\begin{tabular}{lllllll}
\hline & $\begin{array}{l}\text { Number of the } \\
\text { individual } \\
\text { indicators }\end{array}$ & $\begin{array}{l}\text { Rank } \\
\text { Sub-index }\end{array}$ & $\begin{array}{l}\text { Rank } \\
14-30\end{array}$ & $\begin{array}{l}\text { Rank } \\
31-60\end{array}$ & $\begin{array}{l}\text { Rank } \\
61-100\end{array}$ & $\begin{array}{l}\text { Rank } \\
101-133\end{array}$ \\
\hline $\begin{array}{c}\text { Resource Environment } \\
\quad \text { Competitiveness }\end{array}$ & 14 & 0 & 0 & 1 & 10 \\
$\begin{array}{c}\text { Ecological Environment } \\
\quad \text { Competitiveness }\end{array}$ & 11 & 2 & 1 & 3 & 3 & 2 \\
$\begin{array}{c}\text { Environment Carrying } \\
\quad \text { Competitiveness }\end{array}$ & 15 & 0 & 2 & 1 & 3 & 9 \\
$\begin{array}{c}\text { Environment Management } \\
\quad \text { Competitiveness }\end{array}$ & 10 & 3 & 0 & 2 & 3 & 1 \\
$\begin{array}{c}\text { Environment Harmony } \\
\quad \text { Competitiveness }\end{array}$ & 10 & 0 & 1 & 3 & 2 & 4 \\
Total & 60 & 8 & 4 & 9 & 12 & 26 \\
\hline
\end{tabular}

Open Access This chapter is distributed under the terms of the Creative Commons Attribution Noncommercial License, which permits any noncommercial use, distribution, and reproduction in any medium, provided the original author(s) and source are credited. 